\title{
Interactive comment on "Status of the Tibetan Plateau observatory (Tibet-Obs) and a 10-year (2009-2019) surface soil moisture dataset" by Pei Zhang et al.
}

Mirko Mälicke (Referee)

mirko.maelicke@kit.edu

Received and published: 6 November 2020

The presented manuscript "Status of the Tibetan Plateau observatory (Tibet-Obs) and a 10-year (2009-2019) surface soil moisture dataset" by Zhang et al. describes an interesting dataset of in situ soil moisture measurements and an upscaled data product.

Long-term soil moisture datasets are important to foster our understanding of aboveground and below-ground hydrological processes that take place on a multitude of temporal and spatial scales. The actual dataset is available in a data repository with $\mathrm{DOI}$ and presented in a clear structure with a lot of auxiliary tables and descriptions. But, from my point of view, the manuscript is also showing some moderate to major 
issues that need to be resolved before publication. The methods need to shift their focus from upscaling methods to more complete data and study site descriptions. In addition, the referenced data source seems to be incomplete. Detailed comments are listed below, grouped into my main points, comments on the data source itself, minor comments, and a few technical points.

With these issues addressed, I am looking forward to see this really interesting work published.

With kind regards,

Mirko Mälicke

\section{main comments}

These are my main comments in the order of importance:

1. The authors reference different data sources in the manuscript: precipitation data from the China Meteorological Data Service Center (p.5 I.153) and three different model-based soil moisture products (p.5 I.163; p.5 I.171; p.5 I.175). In all three cases, the data is referenced by an URL, without a proper entry in the bibliography or footnotes. Important information, like the access date, is missing.

Beyond this, I have some very serious issues with referencing the landing page of a national authority as a data source. It is not obvious, where data can be downloaded. Further, the search for the given station Maqu did not return any result and I am missing crucial information like the dataset name or aggregation level, which can be specified on the website. The precipitation data is absolutely necessary to reuse the dataset and apply the methods as presented. Thus I 
would strongly recommend adding a copy of the data that has actually be used to the data repository. Neither the data repository nor the manuscript gives any metadata about precipitation (except a rough location).

The same applies to the model-based soil moisture products. In all three cases, the given $U R L$ references the landing page of the respective product, not the data. The GLDAS website still had a maintenance notice from September 2020 (writing on 05.11.2020) and is down. This highlights the importance of adding all the needed data to the repository.

2. From my point of view, a proper study site description and measurement setup is missing and should be added to the manuscript. The dataset itself gives some very broad information like hill top or desert, but that does not really foster my understanding of the conditions at the sites. Maybe there are even images of the study sites available?

The following questions might be helpful to be considered:

How does the terrain (especially aspect and slope) look like at each site?

What are the meteorological conditions at each site?

What is the landuse?

How were the sensors calibrated?

Which steps have been undertaken to check the data quality?

Were there corrections applied to ensure data quality?

Was the measuring interval set to 15 minutes or was the raw data aggregated to 15-minute intervals?

Does the device integrate over the given period, or does it sample the soil moisture every 15 minutes? What are the soil types at the sites, or the geology?

What are the general hydrologic conditions on the sites? Are there rivers nearby? Are the sites in a wet, semi-arid or even arid climate?

Is there possible influence groundwater on the SM measurements?

Is there something else measured at or nearby the sites? Are any helpful atmo-

ESSDD

Interactive

comment 
spheric data available that could be included?

Have there ever been any laboratory measurements of probes from the sites, like

soil density, tension, porosity, or water content measurements?

Is soil profile data available? Maybe even images?

Why was that data recorded in the first place?

Interactive

What does the specific setup at one site look like?

comment

The same applies to all other data products that are used.

From my understanding, the manuscript should, as a dataset description paper, put more focus on a description of the study sites and the data itself, and less focus on a comparison of upscaling methods.

3. The authors present the AA method (p.6 I.193-196) as the prime method to upscale soil moisture measurements. It is hard to believe for me personally, that this approach is really used in recent studies to create high-quality upscaled soil moisture products. A few references of recent studies would be most helpful for me.

Further, the manuscript compares different upscaling methods to each other, but not all upscaled data products are included in the dataset. From my understanding, a method comparison can be published in a scientific method development paper (and then be referenced here). In this dataset description paper, I find it only useful as auxiliary or additional information to the actual dataset as long as all the data (of the other methods) along with the necessary metadata is included.

Finally, if I didn't misunderstand the method, I cannot see how the arithmetic mean of about 20 single values per timestep is useful as a dataset of its own. This processing step can easily be reproduced without any effort. It is presented as the main and core data product of this publication. I would strongly recommend to clarify this point and put the focus on the actual measured data. This 
includes shifting the focus from upscaling methods to data and study site descriptions.

4. I would like to comment on the part p. I.267-270. The authors use the CEC, which is only described in the appendix, to chose the best upscaling method. The selected upscaling product is then used as ground truth to assess all upscaling methods. On the one hand, the CEC is itself the core of the TS upscaling method, which is problematic as the CEC as an objective function for selection is then not objective anymore. On the other hand, the AA upscaling results are selected as ground truth, which is problematic as all four upscaled data products, including the $A A$ results, are assessed using the ground truth dataset (p.9 I.285-287). At the same time, only the AA upscaled data is included in the dataset, not the others. My personal expectation of ground truth included in a data publication, would be independent measurements, that can be used as an objective reference.

I would strongly recommend clarifying the process of upscaling the dataset and include all data.

5. Most of section 4.3 (p.10 I.343 - p.11 I.363) compares the different model-based soil moisture products with the upscaling result that is considered to be the ground truth. Unfortunately, it was not possible to download all products due to server maintenance and unclear sources (as described in point 1) to directly compare any of the products.

The model-based products come at different spatial and temporal scales than the measuring network. To me, it is not clear, which of both over- or underestimates the SM variability. Maybe a comparison of all upscaled products, or even better: SM measurements, to all model-based products and less a validation of one through the other, would be more insightful to better classify the presented data in terms of their expressiveness for SM variability.

Interactive 
In any case, the trend analysis (section 4.2) and its application to model-based products (section 4.3) need to be better connected to the actual measurements presented in the dataset. For me, it would be helpful to better point out, how this information is useful for understanding or using the data.

\section{The dataset}

These comments concern only the dataset itself given at https://doi.org/10.4121/uuid:21220b23-ff36-4ca9-a08f-ccd53782e834

1. The most important part is already mentioned in the main comments, point 1. I would strongly encourage the authors to include all used data into the data repository.

2. I think the dataset should be enriched with some more metadata. E.g. which sensor was used? What is the unit? How precise are the measurements? How does the study site look like? How does the terrain look like?

Please also refer to my main comment 2.

3. It would be most helpful to include the soil temperature and electric conductivity data recorded by the 5TE into the dataset. That would tremendously foster the re-usability of the dataset. In addition, is there a specific reason why the other depths are not included?

4. Please consider extending the dataset description on the source. E.g. include the upscaling method used, name the sensors used or give more details on the context and catchment the sites are operated in. The data source itself should contain all necessary information to use the data. The authors could reuse many parts of this paper here. 
As a personal remark, I found Table 1 of this publication really useful. Together with an overview plot of all measurements, it could be added to the user information PDF as well.

5. The manuscript reports on four different upscaling methods, but only one upscaled result is included in the source. I would suggest to include the other upscaled products and model-based products, as well.

Interactive

comment

6. minor: Please consider using an open data format like ODS, CSV or HDF5 instead of XLSX. The relevant software needed to open XLSX is neither free nor open.

\section{minor comments}

These are my minor comments, in order of appearance:

1. p.2 I.73-74: "apparent-thermal-inertia (ATI) method."; It would be most helpful to reference the actual implementation of this method, as the application ranges from surface heterogeneity analysis on Mars to soil moisture monitoring.

2. p.3 I.76: "different spatial upscaling methods has been scarcely assessed"; Without being an expert on that field, I would like to name studies like Qui et al. (2013), Wang et al. (2014, 2015), Gao et al. (2017) or Moghaddam et al. (2014), which to my understanding, assess upscaling methods for in situ soil moisture measurements. I would suggest clarifying if and why the named approaches might not be suitable for an application in the Tibet-Obs.

3. p.3 I.84-86: Why exactly were the named methods (AA, VD, TS, ATI) chosen? Are there any specific reasons, that make them most suitable? Is there a reason to exclude geostatistical approaches for upscaling? 
4. p.3 I I.85-86: "In addition, the Mann-Kendall (M-K) analysis is adopted to analyse the trend of both regional-scale SM and precipitation time series."; For me, this sentence falls a bit out of context and is neither connected to the previous nor the following sentences. Why a trend analysis? Why precipitation? What kind of precipitation data? Why a M-K test that only tests on monotone trends?

Interactive

comment

5. section 2.1: throughout the whole section 2.1, the authors introduce the different sites and networks. I was asking myself, what exactly is a site, what makes up a network? Is a site a single soil profile? Are networks just sites close to each other, or do they share common ground like a catchment, landuse or similar? I would suggest adding a short definition of these terms.

6. p.7 I.229: " $\Theta_{t}^{t r u}$ represents the reference $S M$ that is considered as the ground truth"; What is the reference SM here? To my understanding, ground truth is an independent measurement of the modeled variable, which has not been described as part of the networks so far. Please clarify.

7. p.8 I.263-I.270: This paragraph references the method MRD, CEC, and $\sigma(R D)$, which are not described in the method part. Please describe, define, and add them.

8. p.10 I.321-324: The authors describe that some sites where damaged and new ones were built in an area of distinctly different conditions. I would like the authors to elaborate on how this is expected to affect a monotonic trend analysis, like $\mathrm{M}-\mathrm{K}$, and what exactly the reader can thus learn from this analysis.

Taking p.10 .329-334 into account, to me it seems like the long-term trends in the data are very much dependent on the locations of the sites and not caused by any other driver, like changing meteorological conditions. I think the trend analysis is helpful for understanding the dataset, but more from a data quality perspective, than to describe the study site (like on p.10 I.338-341). I would consider a rework 
of the whole section 4.2 , to better highlight why the trend analysis was done and what the conclusions and implications for using the dataset are.

9. Discussion: From my point of view, major parts of the discussion need substantial revisions. The whole paragraph p.12 I.387 - 403 remains quite unclear to me. It is referring to Nine levels of RMSE standard (p.12 I.393), which are not further described in the methods. Please clarify what that is. Subsequently, different percentages of these levels are reported and discusses. From these numbers, it is concluded to shut a number of sites down. Why?

In the following paragraph p.12 I.404-418 this is applied to the networks but I honestly don't get the point of this paragraph. Why would you want to decrease the number of sites? What is a "Best level of RMSE" (I.412)? What does "all the possible combinations" (I.413-414) refer to and what is $C_{17}^{12}=6188$ ? What is "the $100^{\text {th }}$ combination of monitoring sites" (I.417-418) ?

10. Figure 1 (p.19): I would strongly recommend reworking this figure. The overview of all sites is of crucial importance to understand the dataset. Please consider including an overview map of Eastern Asia or China, to let the reader better locate the networks. Please consider using larger symbols and especially enlarge the CMA weather station symbols. It would also be helpful to include some larger cities into the maps if there are any. That will make the orientation easier. Maybe it's even a good idea to split this figure into multiple maps and add information like geology or landuse to the maps, if available.

11. Finally, I really liked Table 1 . Here the authors collected studies that were conducted on the different study sites, the data used, and key findings. I would consider briefly describing some of the most interesting findings in the introduction.

Interactive

comment 
1. I would suggest to actually include the caption to Fig.6, instead of referencing another figure's caption.

2. p.2 I.66: "Studies have also reported..."; It is a bit unclear for me, which studies the authors are referring to. The two by Su (sentence before), or the ones named in the following paragraph (Colliander, Quin, Dente...)?

3. p.2 I. 74-75: "several particular or alternative methods [...] were adopted in some investigations."; I would suggest to either specify the actual methods and investigations or to drop the sentence.

4. p.3 I.79-83: The authors introduce their SM network here. I would suggest referencing Fig. 1 here, to point the reader to the overview map. This makes the whole section way clearer.

5. p.4 I.111-112; I.126-127; I.141: here, the authors specify the soil texture for the different SM networks. Please clarify why only the soil texture is specified and not other soil properties? How is that expected to influence the measurements and the quality of the dataset? Where does this information come from?

6. p.4 .143: Just out of curiosity: How can you install a 5 TE at $2.5 \mathrm{~cm}$ depth? Can you comment on data quality here?

7. p.5 I.146 "only the Naqu and MS have collected"; what is MS referring to here?

8. p.5. I.151 "The daily precipitation from two weather stations (Fig. 1)"; I think the symbols on the map are way too small. I can't really see the weather stations in Fig.1

9. p.5 I.158 What is the ECMWF? 
10. p.5 I.161 What is H-TESSEL and CY45R1?

11. p.7 I.210: "the average SM data"; What is the average SM data? I am not sure what average is referring to here.

12. p.8 I.267: If Eq. (A3) gives the bias of the upscaled product, what does Eq. (3a) then calculate? Is it the same, or another bias? Please clarify.

13. p.9. I.311 - p.10 I.312: With an extent of 10 years on the $x$-axis, it is very hard for me to see the described differences in value. I can hardly see the data. Please consider adding another plot or subplot with a shorter time span.

\section{References}

Gao, Shengguo, et al. "Upscaling of sparse in situ soil moisture observations by integrating auxiliary information from remote sensing." International Journal of Remote Sensing 38.17 (2017): 4782-4803.

Moghaddam, Mahta, et al. "The SoilSCAPE network multiscale in-situ soil moisture measurements: Innovations in network design and approaches to upscaling in support of SMAP." AGUFM 2014 (2014): IN11A-3599.

Qin, Jun, et al. "Spatial upscaling of in-situ soil moisture measurements based on MODIS-derived apparent thermal inertia." Remote Sensing of Environment 138 (2013): $1-9$.

Wang, Jianghao, et al. "Upscaling in situ soil moisture observations to pixel averages with spatio-temporal geostatistics." Remote Sensing 7.9 (2015): 11372-11388. 
Wang, Jianghao, et al. "A geostatistical approach to upscale soil moisture with unequal precision observations." IEEE Geoscience and Remote Sensing Letters 11.12 (2014):

ESSDD 2125-2129.

Interactive comment on Earth Syst. Sci. Data Discuss., https://doi.org/10.5194/essd-2020-209, 2020.

Interactive

comment 